\title{
Stimulus intensity-dependent modulations of hippocampal long-term potentiation by basolateral amygdala priming
}

\author{
Zexuan $\mathrm{Li}^{1+}$ and Gal Richter-Levin ${ }^{1,2,3 *}$ \\ 1 Institute for the Study of Affective Neuroscience, University of Haifa, Haifa, Israel \\ ${ }^{2}$ Department of Neurobiology, University of Haifa, Haifa, Israel \\ ${ }^{3}$ Department of Psychology, University of Haifa, Haifa, Israel
}

\section{Edited by:}

Nicola Maggio, The Chaim Sheba

Medical Center, Israel

Reviewed by:

Oliver Stork, Otto von Guericke

University Magdeburg, Germany

Wickliffe C. Abraham, University of

Otago, New Zealand

\section{*Correspondence:}

Gal Richter-Levin, Department of

Psychology, University of Haifa,

Haifa 31905, Israel.

e-mail: galrichterlevin@gmail.com

${ }^{\dagger}$ Present address:

Mental Health Institute of the 2nd Xiangya Hospital, Central South

University, Changsha, Hunan,

People's Republic of China.
There is growing realization that the relationship between memory and stress/emotionality is complicated, and may include both memory enhancing and memory impairing aspects. It has been suggested that the underlying mechanisms involve amygdala modulation of hippocampal synaptic plasticity, such as long-term potentiation (LTP). We recently reported that while in CA1 basolateral amygdala (BLA) priming impaired theta stimulation induced LTP, it enhanced LTP in the dentate gyrus (DG). However, emotional and stressfull experiences were found to activate synaptic plasticity within the BLA, raising the possibility that BLA modulation of other brain regions may be altered as well, as it may depend on the way the BLA is activated or is responding. In previous studies BLA priming stimulation was relatively weak ( $1 \mathrm{~V}, 50 \mu \mathrm{s}$ pulse duration). In the present study we assessed the effects of two stronger levels of BLA priming stimulation ( $1 \mathrm{~V}$ or $2 \mathrm{~V}, 100 \mu \mathrm{s}$ pulse duration) on LTP induction in hippocampal DG and CA1, in anesthetized rats. Results show that 1V-BLA priming stimulation enhanced but 2V-BLA priming stimulation impaired DG LTP; however, both levels of BLA priming stimulation impaired CA1 LTP, suggesting that modulation of hippocampal synaptic plasticity by amygdala is dependent on the degree of amygdala activation. These findings suggest that plasticity-induced within the amygdala, by stressful experiences induces a form of metaplasticity that would alter the way the amygdala may modulate memory-related processes in other brain areas, such as the hippocampus.

Keywords: emotional memory, plasticity, stress, limbic system, amygdala, rat

\section{INTRODUCTION}

It is generally accepted that memory is organized in multiple brain systems that can functionally interact with each other (Squire and Zola, 1996; Thompson and Kim, 1996). Two of these specialized systems are the hippocampus, which is crucial for associative type (Kim and Fanselow, 1992; Phillips and LeDoux, 1992; Morris, 2001; Day et al., 2003) and other types of learning and memory (Morris et al., 1982; Eichenbaum et al., 1996; Squire and Zola, 1996; Abrahams et al., 1999), and the amygdala, which plays a pivotal role in mediating many aspects of the stress response, fearmotivated learning, and memory for emotionally evocative events (LeDoux, 2000, 2003; McGaugh, 2004; Berretta, 2005). It has been suggested that emotional arousal/stress activates the amygdala and that this activation, specifically that of the basolateral amygdala (BLA), results in modulation of memory-related processes in the hippocampus (McGaugh, 2000; Richter-Levin and Akirav, 2000; Roozendaal, 2000; Packard and Cahill, 2001; Richter-Levin, 2004; LaBar and Cabeza, 2006). Thus, these two structures form a functional system relevant to the complicated effects of emotionality and stress on learning and memory (Kim and Diamond, 2002; Roozendaal, 2002; Prickaerts and Steckler, 2005; Lupien et al., 2007). On the one hand, considerable research shows that the interactions between amygdala and hippocampus are necessary for the enhanced encoding and consolidation of memory for emotionally arousing material and contexts (Cahill, 2000; Canli et al., 2000; Packard and Cahill, 2001; Phelps, 2004; Phelps and LeDoux, 2005). On the other hand, amygdala activation is suggested to mediate stress-induced impairment of hippocampusdependent memory (Akirav and Richter-Levin, 2006; Hurlemann et al., 2007a,b; Maroun and Akirav, 2008).

Consistent with the complex relationship between stress and memory, stress can differently influence synaptic plasticity, such as long-term potentiation (LTP) (Bliss and Collingridge, 1993; Malenka and Nicoll, 1999; Martin et al., 2000; Malenka and Bear, 2004). In the hippocampus, data so far suggest differential susceptibility to stressful events in its different subregions. Extensive observations from in vitro and in vivo electrophysiological studies indicate that variant stress paradigms, including unpredictable and inescapable restraint-tail shock (Foy et al., 1987; Kim et al., 1996; Garcia et al., 1997; Shors et al., 1997) or footshock (Shors et al., 1989; Li et al., 2005), forced exposure to brightly lit and unfamiliar chambers (Diamond et al., 1990; Xu et al., 1997), unavoidable exposure to a predator (Mesches et al., 1999), or platform stress (Maroun and Richter-Levin, 2003), discriminatory avoidance learning (Izaki and Arita, 1996), as well as contextual fear conditioning (Sacchetti et al., 2002; Hirata et al., 2009), all impaired LTP in the CA1. However, different stressors were reported to either impair (Diamond and Rose, 1994; Shors and 
Dryver, 1994; Wang et al., 2000; Korz and Frey, 2005; Ahmed et al., 2006), enhance (Izaki and Arita, 1996; Gerges et al., 2001; Kavushansky et al., 2006; Spyrka and Hess, 2010), or have no effect (Bramham et al., 1998; Garcia, 2001; Gerges et al., 2001; Pavlides et al., 2002; Yamada et al., 2003; Vouimba et al., 2004; Yarom et al., 2008) on the ability to induce LTP in the DG. Furthermore, Korz and Frey (2003) reported a bidirectional effect of behavioral stress on the maintenance of DG-LTP, i.e., handling 15 min after the induction of early LTP resulted in an impairment of LTP, whereas a 2 min swim also 15 min after induction resulted in prolongation of LTP up to $24 \mathrm{~h}$ (Korz and Frey, 2003). Given critical role of amygdala in stress, one can suggest a possible way to explain complex effects of stress on hippocampus-dependent learning and memory that invokes differential effects of stressors on amygdala action, which inturn results in differential effects on LTP in different subregions of the hippocampus (Tsoory et al., 2008).

Indeed, the amygdala is critical in the modulation of hippocampal synaptic plasticity. Lesions of amygdala or pharmacological intervention of its function effectively prevented the effects of stress on hippocampal CA1 LTP, as well as on hippocampusdependent spatial and fear memory (Kim et al., 2001, 2005; Goosens and Maren, 2004; Yang et al., 2008). Also, the behavioral or motivational reinforcement of hippocampal DG LTP (that can transform early LTP into late LTP) are reported to depend on the function of the amygdala (Almaguer-Melian et al., 2003; Korz and Frey, 2005). Consistently, hippocampus DG/CA1 LTP can be modulated by electrical stimulation of the amygdala (Akirav and Richter-Levin, 1999a,b; Abe, 2001; Frey et al., 2001; Akirav and Richter-Levin, 2002; Nakao et al., 2004; Vouimba and Richter-Levin, 2005; Vouimba et al., 2007). However, the profiles of amygdala modulation of hippocampal LTP are intriguing. Thus, while BLA lesions or its pharmacological suppression were reported to impair in vivo LTP of perforant path (PP)-DG (Abe, 2001), Korz and Frey (2005) reported facilitation of DG LTP in BLA lesioned animals (Korz and Frey, 2005). Similarly, BLA stimulation was reported to augment LTP in PP-DG pathway (Ikegaya et al., 1995, 1996; Akirav and Richter-Levin, 1999a; Frey et al., 2001), whereas later reports demonstrated that this effect vary considerably depending on the interplay of the strengths and timing of BLA and PP stimulation (Akirav and Richter-Levin, 1999b; Nakao et al., 2004; Vouimba and Richter-Levin, 2005). For example, although it was reported that BLA stimulation only affects weak and transient forms of LTP and LTP produced by a strong tetanus does not require the BLA for its induction nor its maintenance (Ikegaya et al., 1995; Frey et al., 2001), our previous series of results showed that BLA activation, applied 30s before or $1-2 \mathrm{~h}$ after strong tetanus application, could enhance or impair DG LTP induced by a strong tetanus, respectively, (Akirav and Richter-Levin, 1999a,b, 2002). Furthermore, consistent with our previous findings, we have recently showed that BLA activation (applied $30 \mathrm{~s}$ prior to or after the presentation of stimulation tetanus) impairs CA1 LTP in response to weak tetanus but enhances DG LTP in response to both weak and strong tetanus (Vouimba and Richter-Levin, 2005). Thus, we have suggested that, depending on how the amygdala is activated in terms of intensity, timing relationship, duration, and contextual input during information processing, the hippocampal outcome would involve the components of both/either enhancing and/or suppressing effects (Akirav and Richter-Levin, 2006; Tsoory et al., 2008), possibly providing synaptic plasticity mechanism underlying manifold/heterogeneous effects of stress on memory.

The present study was undertaken to further characterize the intriguing profiles of modulation of hippocampal synaptic plasticity by amygdala in order to elaborate on the mechanisms underlying the complex stress-memory relationship. We have previously demonstrated that relatively weak BLA activation $30 \mathrm{~s}$ prior to LTP induction in the hippocampus enhanced theta stimulation-induced LTP in DG, but decreased LTP in CA1 of the hippocampus (Vouimba and Richter-Levin, 2005). Here, we assessed the effects of two stronger levels of BLA priming stimulation on LTP, induced in hippocampal dentate gyrus (DG) and CA1 subregions in the anesthetized rat. The results demonstrate that BLA priming differently modulates subsequent LTP in the hippocampus depending on the degree of BLA activation.

\section{MATERIALS AND METHODS SUBJECTS}

The experiments were performed using male Sprague-Dawley rats (Harlan Laboratories, Jerusalem, Israel) weighing 270-340 g. Rats were housed in Plexiglas cages (six rats per cage) and were maintained on a free-feeding regimen with a $12: 12 \mathrm{~h}$ light/dark schedule. All electrophysiological testing was performed at least 1 week after their arrival, during the light phase of the cycle. During the course of the experiment, body temperature was monitored and maintained at $37 \pm 0.5^{\circ} \mathrm{C}$ using a regulated heating pad.

All procedures and tests were approved by the Institutional Animal Care Committee and adhered to the guidelines of the NIH Guide for the Care and Use of Laboratory Animals.

\section{ELECTROPHYSIOLOGY \\ Surgery}

Rats were anesthetized $(40 \%$ urethane $+5 \%$ chloral hydrate, $0.5 \mathrm{ml} / 100$ g, i.p.) and mounted on a stereotaxic frame (Stolting, Wood Dale, IL). The scalp was incised and retracted, and the head position was adjusted to place bregma and lambda in the same horizontal plane. Small burr holes (1.5-2 mm diameter) were drilled in the skull for the placement of stimulating and recording electrodes. A reference electrode consisting of a $125 \mu \mathrm{m}$ coated wire was affixed to the skull in the area overlapping the nasal sinus. A recording glass electrode (tip diameter, 2-5 $\mu \mathrm{m}$; filled with $2 \mathrm{M} \mathrm{NaCl}$ ) was stereotaxically positioned in the CA1 pyramidal cell layer [ $4.0 \mathrm{~mm}$ posterior to bregma (AP), $2.5 \mathrm{~mm}$ from midline (ML) and $\sim 2 \mathrm{~mm}$ dorsoventral (DV) from dura] or in the DG granular cell layer $(4.0 \mathrm{~mm} \mathrm{AP,} 2.5 \mathrm{~mm} \mathrm{ML}$ and $2.7-3.0 \mathrm{~mm}$ DV from dura). Bipolar concentric stimulating electrodes ( $125 \mu \mathrm{m}$; Kopf, Tujunga, CA) were inserted in the ipsilateral BLA (2.8 mm AP, $4.8 \mathrm{~mm} \mathrm{ML}$, and $7.6 \mathrm{~mm} \mathrm{DV})$ and either in the contralateral ventral hippocampal commisure (vHC: $2 \mathrm{~mm} \mathrm{AP}, 1.5 \mathrm{~mm} \mathrm{ML}$, and $\sim 3 \mathrm{~mm}$ DV from dura) for activating field potentials in the CA1 or in the ipsilateral perforant path (PP: $8 \mathrm{~mm}$ AP, $4.0 \mathrm{~mm} \mathrm{ML}$, and 2.5-3.0 $\mathrm{mm} \mathrm{DV}$ from dura) for 
activating field potentials in the DG. The DV location of the recording and stimulating electrodes was adjusted to maximize the amplitude of evoked field potentials.

\section{Stimulating and recording procedures}

CA1 and DG field potentials evoked by single pulses delivered to the $\mathrm{vHC}$ or $\mathrm{PP}$, respectively, $(0.1 \mathrm{~ms}$ rectangular monophasic pulses) were amplified $(\times 1000)$ (AM-Systems amplifier), displayed on an oscilloscope, digitized at $10 \mathrm{kHz}$ (CED) and stored to disk for off-line analysis (Spike-2 software). Baseline responses were established by means of a stimulation intensity sufficient to elicit a response representing $20-40 \%$ of the maximal amplitude of the evoked-field potentials. LTP was assessed by measuring both the increase in the population spike amplitude (PS) and the slope of the excitatory postsynaptic potential (EPSP) component, for the DG. However, in CA1, because of the early occurrence of the PS in some recordings, the slope was not measurable and, therefore, only the PS was analyzed and reported here. In our previous study (Vouimba and RichterLevin, 2005) we have shown that both the PS and the slope of the EPSP (for both DG and CA1) follow the same pattern of changes responding to theta stimulation and amygdala modulation.

\section{Protocols}

Stable baseline recording of evoked-field potential in DG or CA1 was established for $30 \mathrm{~min}$ ( 1 pulse every $15 \mathrm{~s}$ ) for all groups [For both DG and CA1: Control (electrode placed in the BLA but not stimulated), BLA Priming (1 V), and BLA Priming (2 V)]. Following baseline recording, LTP was induced by moderate theta burst stimulation (mTS: 10 trains, each consisting of 10 pulses at $100 \mathrm{~Hz}$, with and intertrain interval of $200 \mathrm{~ms}$; with trains delivered at test stimulus intensity) relative to our previously used strong TS (three sets of 10 trains; each train consisted of 10 pulses at $100 \mathrm{~Hz}$, with an intertrain interval of $200 \mathrm{~ms}$ and an interset interval of $1 \mathrm{~min}$ ) and weak TS (one set of five trains, each train consisting of five pulses at $100 \mathrm{~Hz}$, with an inter-train interval of $200 \mathrm{~ms}$ ) to the PP or vHC (Akirav and Richter-Levin, 1999a,b, 2002; Vouimba and Richter-Levin, 2005; Vouimba et al., 2007). After mTS stimulation, responses to test pulse stimuli were recorded every $15 \mathrm{~s}$ for $1 \mathrm{~h}$. The BLA Priming groups received a priming stimulation of BLA ( $1 \mathrm{~V}$ or $2 \mathrm{~V}, 100 \mu \mathrm{s}$ pulse duration, 10 trains of five pulses at $100 \mathrm{~Hz}$; intertrain interval, $200 \mathrm{~ms}) 30 \mathrm{~s}$ before mTS to the PP or $\mathrm{vHC}$ was applied. The $1 \mathrm{~V}$ BLA priming stimulation ( $1 \mathrm{~V}, 50 \mu$ s pulse duration, 10 trains of five pulses at $100 \mathrm{~Hz}$; intertrain interval, $200 \mathrm{~ms}$ ) used in a previous study (Vouimba et al., 2007) had no significant effects on EPSP slope while having a small but significant effect on PS in DG when compared with the naive control; however, it had no effects on both EPSP slope and PS in CA1. In a pilot experiment for the current study, both BLA priming stimuli had no significant effects on EPSP slope in DG of the three tested groups (the two BLA priming groups and the naïve control group); the $1 \mathrm{~V}$ BLA priming stimulus resulted in a small but significant increase of PS in DG compared with the naïve control group, as was found in our previous study. The other stimulus intensity $(2 \mathrm{~V})$ had a small but not significant effect on DG PS and there was no significant difference between the two BLA priming groups. The two intensities of BLA priming stimuli had no effects on EPSP slope or PS in CA1. In addition, at both stimulus intensities, there were no visible responses in DG/CA1 in response to single stimulation pulses to the BLA. The Control animals were implanted in the BLA with stimulating electrodes through which no priming stimulation of BLA was applied.

\section{HISTOLOGY}

After completion of the electrophysiological examination of the recording in DG or CA1, electrical lesions of BLA were made by passing anodal current in stimulating electrodes in BLA group animals ( $5 \mathrm{mV}$ DC for $20 \mathrm{~s}$ ), not including the control animals with BLA stimulating electrodes through which no stimulation was given. Animals were then decapitated and their brains were frozen at $-80^{\circ} \mathrm{C}$ for further analysis. Coronal sections $(60 \mu \mathrm{m})$ were cut using LEICA cryostat, and mounted on glass microscope slides. Sections were stained with Cresyl violet. The stimulating electrodes placements were verified under microscopic examination according to the atlas (Paxinos and Watson, 1997).

\section{STATISTICS}

The amplitude of the PS and the slope of the EPSP were expressed as the mean percentage $( \pm$ SEM) of the individual basal values of animals for each group. Only the animals that had correct positions of the stimulating electrodes in the BLA, according to the histology, were included in the analysis. Student's paired $t$-test was used to decide whether or not LTP was induced after TBS in each group. ANOVA for repeated measures with least significant difference test (LSD) as post-hoc test was used to compare LTP between groups. Statistical significance was set at $P<0.05$.

\section{RESULTS}

\section{HISTOLOGY}

Figure 1 illustrates the location of stimulating electrode site in BLA. Histological analysis revealed that near $90 \%$ of rats recorded had correct positions of the stimulating electrodes in the BLA. Only data from these subjects was used in the analysis.

\section{EFFECTS OF BLA ACTIVATION ON LTP-INDUCED BY MTS IN CA1 AND THE DG}

Paired $t$-test revealed that in all the groups mTS reliably induced LTP of PS and slope in DG and LTP of PS in CA1 [ $t$-tests for difference from baseline $(100 \%)$ at $60 \mathrm{~min}$ after mTS. PS amplitude/EPSP slope in DG: Control group (201\% of baseline, $\left.t_{8}=-6.3, P<0.001\right) /\left(123 \%\right.$ of baseline, $\left.t_{8}=-8.8, P<0.01\right)$; $1 \mathrm{~V}$-BLA priming group $\left(274 \%\right.$ of baseline, $t_{8}=-12.3, P<$ $0.001) /\left(134 \%\right.$ of baseline, $\left.t_{8}=-10.4, P<0.001\right) ; 2 \mathrm{~V}$-BLA priming group $\left(157 \%\right.$ of baseline, $\left.t_{9}=-3.7, P<0.01\right) /(114 \%$ of baseline, $\left.t_{9}=-6.0, P<0.001\right)$. PS amplitude in CA1: Control group $\left(415.5 \%\right.$ of baseline, $\left.t_{6}=-3.8, P<0.01\right)$; $1 \mathrm{~V}$-BLA priming group ( $243 \%$ of baseline, $\left.t_{6}=-5.7, P<0.005\right)$; 2V-BLA priming group $\left(238 \%\right.$ of baseline, $\left.t_{6}=4.5, P<0.005\right)$. See Figures 2A,C and 3A]. ANOVA analysis conducted on both DG LTP and CA1 LTP at different time points [groups $\times$ time 


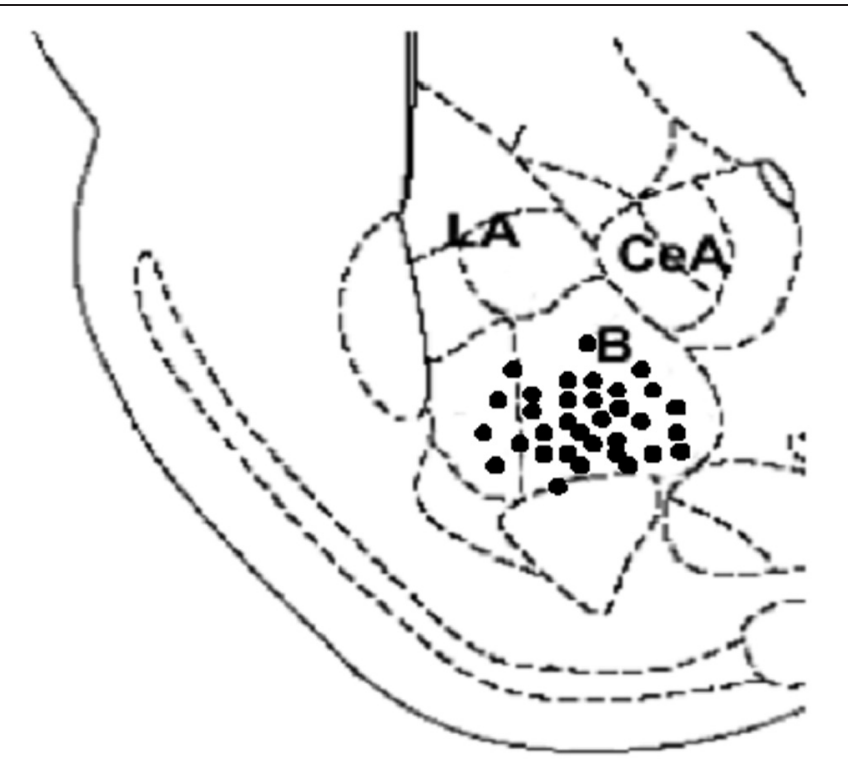

FIGURE 1 | A diagram depicting a coronal section of the rat brain (2.8-3.0 $\mathrm{mm}$ posterior to Bregma) showing electrode placements in the BLA. Filled circles indicate locations for all BLA groups $(n=33 ; \mathrm{B}$, basal amygdala; La, lateral amygdala; CeA, central amygdala).

$(3 \times 12)]$ revealed significant difference between the groups (Control group, 1V-BLA priming group and 2V-BLA priming group) [PS amplitude/EPSPs slope in DG: $F_{(2,25)}=9.96$, $P<0.005 / F_{(2,25)}=5.41, \quad P<0.02$; PS amplitude in CA1: $F_{(2,18)}=3.858, P<0.05$. See Figures 2A,C and 3A].

In DG, 1V-BLA priming (30 s prior to application of $\mathrm{mTS}$ to PP-DG) enhanced LTP compared with Control, showing significantly greater PS-LTP at all time points pos-mTS $(P s<0.05$; See Figure 2B) and significantly greater slope-LTP at the last two 10 min-period blocks ( $P s<0.05$; See Figure 2D). In contrast, 2V-BLA priming (30 s prior to application of mTS to PP-DG) impaired LTP of PS compared with Control, showing significantly smaller PS-LTP $(P<0.05$; See Figure 2B $)$ and significantly smaller slope-LTP $(P<0.05$; see Figure 2D) at the last $10 \mathrm{~min}$ period block post-mTS. Comparison between the two levels of BLA priming activation demonstrated that LTP in DG induced by mTS following 1V-BLA priming activation was significantly different from that induced by mTS following 2V-BLA priming activation for PS at time points post-mTS $(P S<0.01$; See Figure 2B) and for slope at all time points post-mTS except the first initial $10 \mathrm{~min}(P s<0.05$; See Figure 2D).

In contrast, in CA1, the response to BLA priming activation was quite different. In comparison to Control, both levels of BLA priming (30 s prior to application of mTS to vHC-CA1) similarly impaired PS-LTP (1V-BLA priming vs. 2V-BLA priming, $P s>0.05$; see Figure 3), with 2V-BLA priming activation significantly inhibiting PS-LTP in CA1 at all time points postmTS (compared with Control, $P$ s $<0.05$. see Figure 3B) and 1V-BLA priming activation significantly inhibiting PS-LTP in CA1 at the last two 10 min-period blocks post-mTS (compared with Control, $P$ s $<0.05$. see Figure 3B). Thus, BLA activation differentially modulated hippocampal plasticity, depending on both hippocampal subregions and the degree of BLA activation. Figure 4 showed representative analog traces for baseline and the last 10 min-period block post-mTS from Control and BLA priming groups.

\section{DISCUSSION}

We examined the effects of two levels of BLA priming activation on synaptic plasticity in the PP input to the granule cells in the DG, and the $\mathrm{vHC}$ input to the CA1 pyramidal cells of the hippocampus. The results show that $1 \mathrm{~V}$-BLA priming stimulation enhanced but 2V-BLA priming stimulation impaired DG LTP, whereas both levels of BLA priming stimulation impaired CA1 LTP. These results suggest that amygdala modulation of hippocampal synaptic plasticity is dependent both on the degree of amygdala activation and on hippocampus subregion examined.

We previously found that employing a similar pattern of amygdala priming but with a shorter pulse duration $(50 \mu \mathrm{s})$ (weaker priming) enhanced PP-DG LTP induction (Akirav and RichterLevin, 1999a,b, 2002; Vouimba and Richter-Levin, 2005; Vouimba et al., 2007). Our current results with 1V-BLA priming are consistent with those findings. However, further strengthing BLA priming intensity $(2 \mathrm{~V}, 100 \mu \mathrm{s}$ pulse duration) did not further enhance, but on the contrary inhibited DG LTP, suggesting that different degrees of BLA activation may exert bidirectional influence on synaptic plasticity in the DG. Nakao et al. (2004) reported that the effects of amygdala activation on DG synaptic plasticity could range from LTP to LTD depending on the degree and timing of neural activity of the BLA. They showed that strong BLA activation decreased the LTP-LTD crossover threshold to favor LTP, whereas weak BLA activation increased it. Beyond possible differences in the exact parameters/patterns of the BLA and PP stimulation between the two studies, there might exist an inverted U-shape function between the degree of BLA activation and its effects on the DG LTP, resembling that of glucocorticoidhippocampal LTP relationship (De Kloet, 2004). In any case, the results demonstrate that BLA activation may result in enhancing or suppressing effects on DG-LTP, depending on the exact parameters of BLA activation.

In contrast, in the $\mathrm{vHC}$-CA1 pathway, both $1 \mathrm{~V}$-BLA priming and 2V-BLA priming impaired vHC-CA1 LTP. These results are in agreement with previous findings demonstrating that relative weak BLA priming activation attenuated the LTP induction in this pathway (Vouimba and Richter-Levin, 2005; Vouimba et al., 2007). Together, the results suggest that LTP in CA1 is more susceptible to intereference by amygdala activation compared with DG.

Stress hormones, glucocorticoids (GC), and noradrenaline (NA) with their synergistic interaction in the BLA are suggested to mediate negative and positive influence on memory by emotion or stress (McGaugh, 2000; Lupien et al., 2007; Hurlemann et al., 2007a,b), and thus could be implicated in the underlying mechanism of our present findings. In support of this, we have previously demonstrated that BLA stimulation effects on DG-LTP involve both NA and GC (Akirav and Richter-Levin, 2002; Vouimba et al., 2007). Furthermore, despite that NA and $\beta$ adrenergic stimulation has been shown repeatedly to be involved 

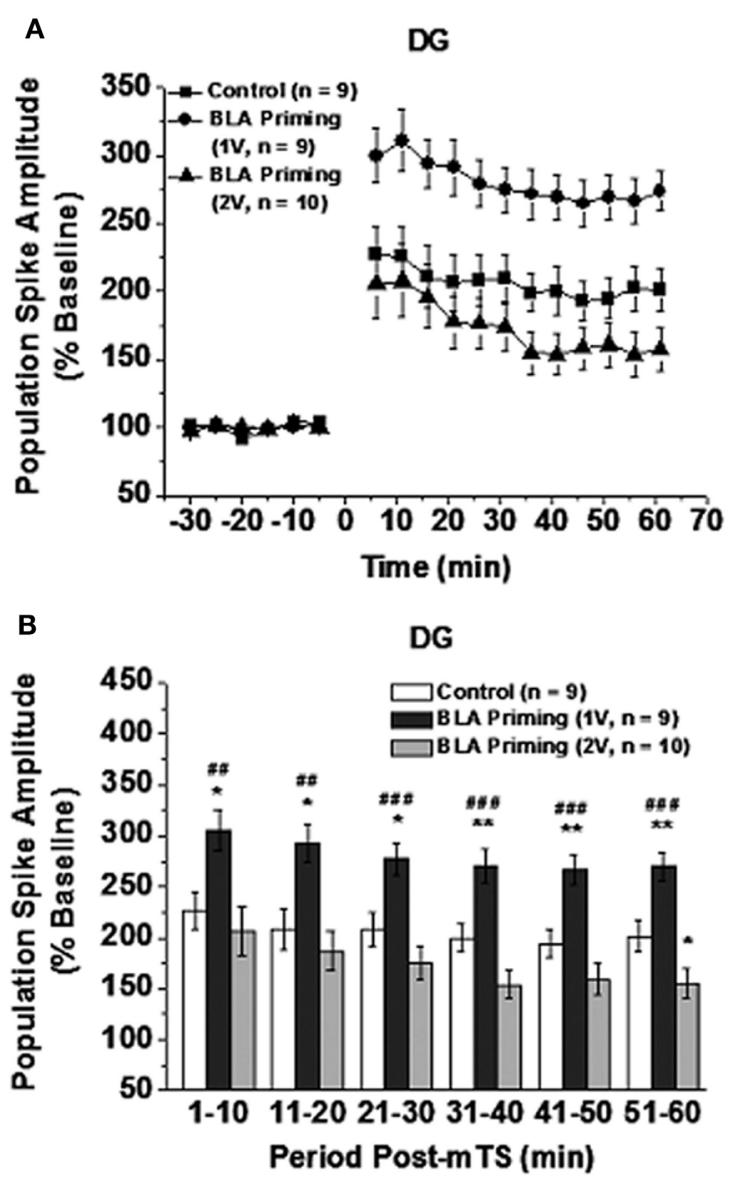

FIGURE 2 | Effect of BLA activation [30 s before moderate theta burst stimulation (mTS: 10 trains, each consisting of 10 pulses at $100 \mathrm{~Hz}$, with an intertrain interval of $200 \mathrm{~ms}$ )] on DG LTP induced by $\mathrm{mTS}$ : Mean ( \pm SEM percentage of baseline). The $\mathrm{mTS}$ reliably induced PS-LTP (A) and slope-LTP (C) with respect to the baseline in all the groups (Paired $t$-Test at $60 \mathrm{~min}$ after mTS, $\left.t_{\mathrm{s}}<-3.70, P_{\mathrm{s}}<0.01\right)$. The $1 \mathrm{~V}$-BLA priming
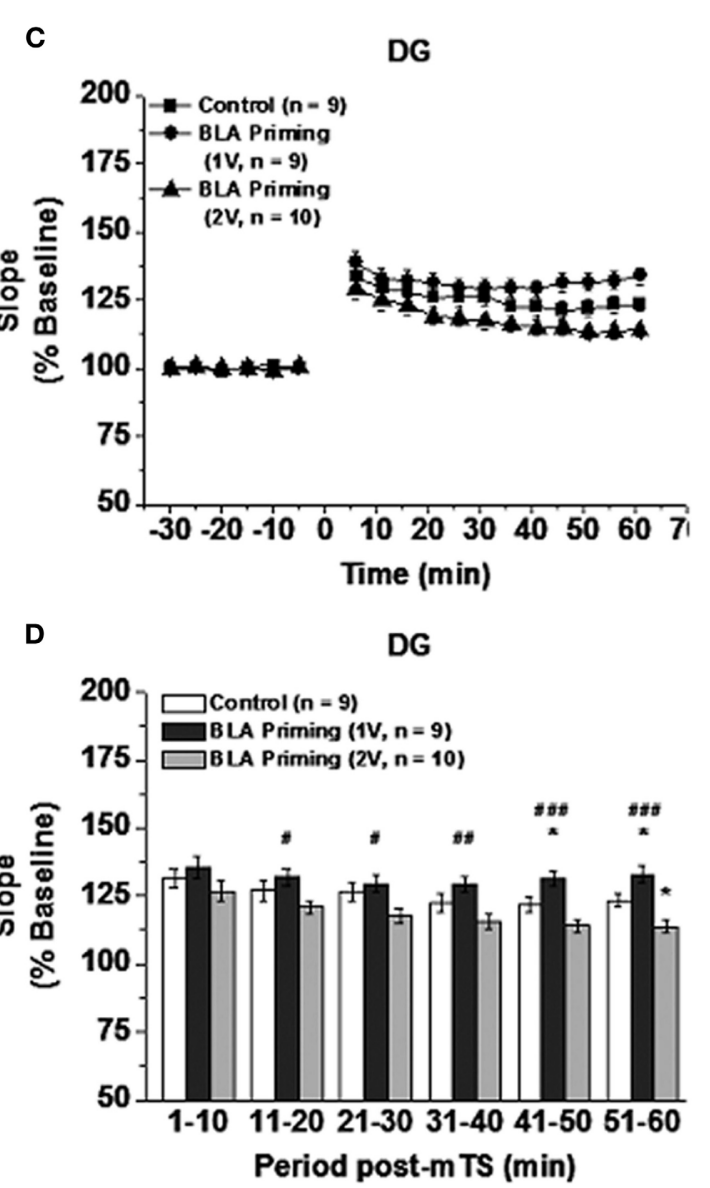

activation enhanced while the $2 \mathrm{~V}$-BLA priming activation impaired the PS amplitude (summarized in $\mathbf{B}$ ) and the slope (summarized in D) of the EPSP component (Two-Way ANOVA with LSD as post-hoc). ${ }^{*} p<0.05$ and ${ }^{* *} p<0.01$ compared with the control; ${ }^{\#} P<0.05$,

\#\# $P<0.01$, and ${ }^{\# \# \# ~} P<0.001$ compared with the 2 V-BLA priming group. in the reinforcement of hippocampal LTP (Izquierdo and Medina, 1995; Thomas et al., 1996; Katsuki et al., 1997; Seidenbecher et al., 1997; Watabe et al., 2000; Gelinas and Nguyen, 2005), recent studies showed that low doses of NA (administered icv) effectively reinforced DG LTP while a higher dose was not effective (Almaguer-Melian et al., 2005). Systematically administered adrenaline enhanced DG LTP in an inverted-U dose-response function (Korol and Gold, 2008), possibly by causing NA release in many brain regions such as hippocampus and amygdala (Gold and van Buskirk, 1978; Williams et al., 1998; Miyashita and Williams, 2004). In contrast to DG LTP, BLA priming effects on CA1 LTP were not affected by blockade of NA or GC activation in BLA (Vouimba et al., 2007), suggesting that the mediating mechanisms of effects differ between CA1 and DG.

On the other hand, connected with stress and stress hormones such as NA showing to activate the amygdala in both humans and animals (Pelletier et al., 2005; Buffalari and Grace, 2009; Roozendaal et al., 2009; van Marle et al., 2009), different stressors may affect amygdala activity in different ways (Roozendaal et al., 2009). For example, Hand et al. (2002) have demonstrated differential release of corticotropin-releasing hormone $(\mathrm{CRH})$ in the amygdala during different types of stressors. The level of activation of the MAP Kinase cascade in the BLA was also found to depend on the level of stress involved (Kogan and RichterLevin, 2008; Ilin and Richter-Levin, 2009). Furthermore, activity in the BLA was found to be enhanced by the application of corticosterone in a dose-dependent manner (Kavushansky and Richter-Levin, 2006). These phenomena support the notion that different patterns of BLA stimulation mimic effects of different stressor-induced alterations in amygdala activation, and such alterations, as indeed demonstrated here, modulate the way the amygdala influences activity and plasticity in other brain regions, such as memory-related processes in hippocampus (Tsoory et al., 2008; Roozendaal et al., 2009).

The amygdala is a pivotal structure associated with stress. It has been suggested before that stress exerts its effects on brain regions, such as the hippocampus, at least partially by activating the BLA (for review: Richter-Levin, 2004 or Tsoory et al., 2008). 


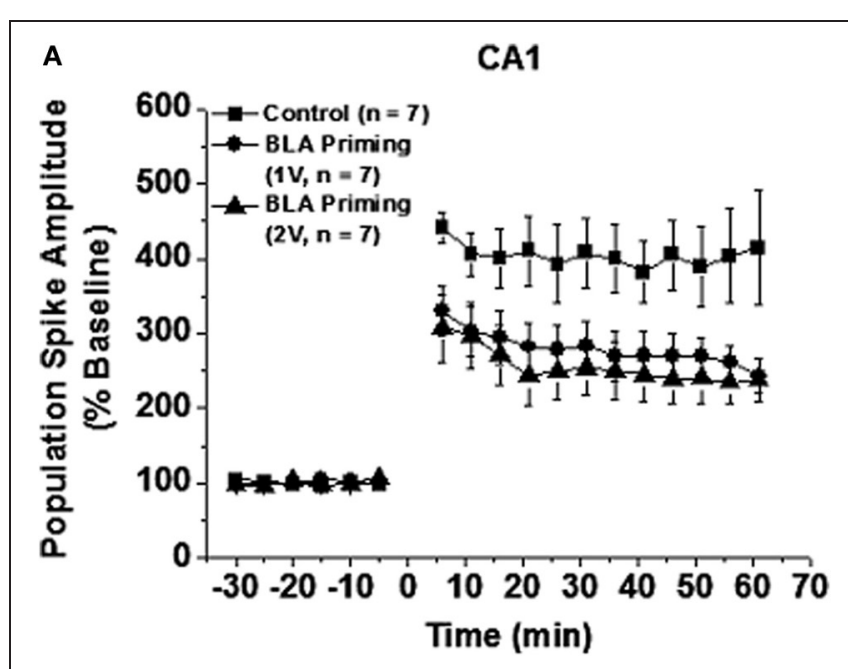

B

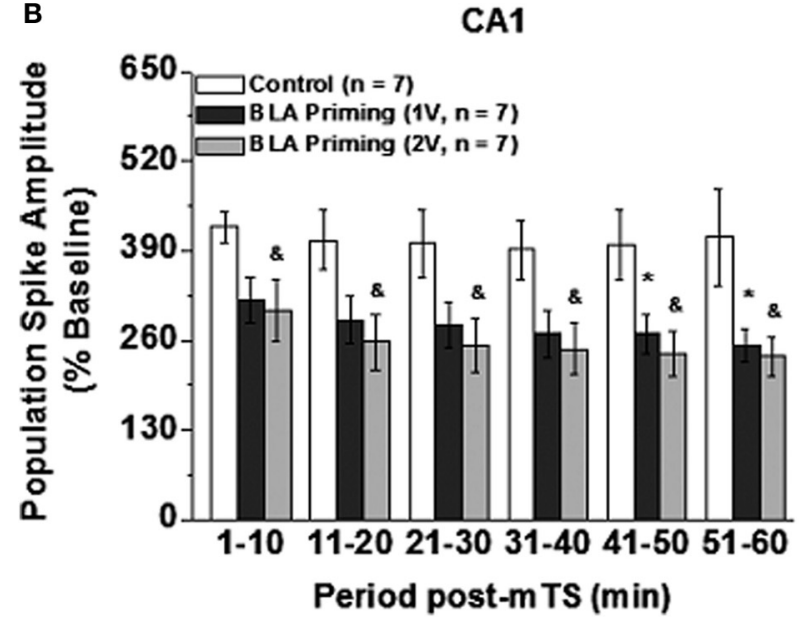

FIGURE 3 | Effect of BLA activation [30 s before moderate theta burst stimulation (mTS: 10 trains, each consisting of 10 pulses at $100 \mathrm{~Hz}$, with an intertrain interval of $200 \mathrm{~ms})$ ] on CA1 LTP induced by mTS: mean ( \pm SEM percentage of baseline). The MTS reliably induced PS-LTP (A) with respect to the baseline in all the groups (Paired $t$-Test at $60 \mathrm{~min}$ after $\left.\mathrm{mTS}, t_{S}<-3.80, P_{S}<0.01\right)$. Both levels of BLA priming activation impaired LTP of PS Amplitude (summarized in B). ${ }^{*} P / \& P<0.05$ compared with the control (Two-Way ANOVA with LSD as post-hoc).

For example, BLA activation induces an increase in serum corticosterone (Rubin et al., 1966; Feldman et al., 1982; Dunn and Orr, 1984). In a recent study (Vouimba et al., 2007), we found that the $1 \mathrm{~V}$ BLA priming stimulation protocol increased serum corticosterone levels to a lower level than that induced by swim stress in a platform-deprived water maze (Kavushansky et al., 2006), though both of these studies showed enhanced LTP in DG and impaired LTP in CA1. However, stronger BLA priming (2V) was found in the present study to impair both DG-LTP and CA1-LTP. This is in agreement with previous findings indicating that while water maze exposure stress enhances DG-LTP (Kavushansky et al., 2006), under-water trauma, which is considered to be a more intense stressor, blocked DG-LTP (Wang et al., 2000). Together, these results indicate that different patterns

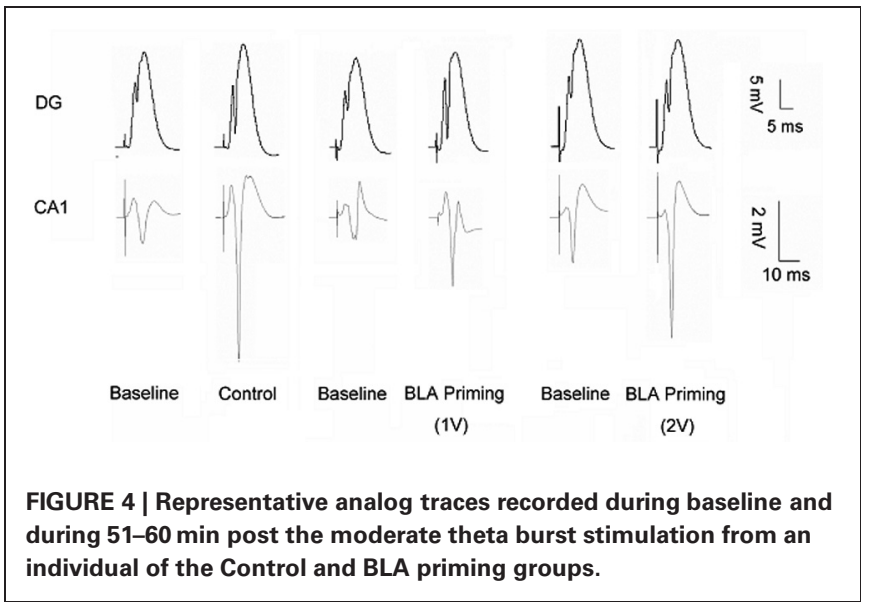

of BLA priming are likely to reflect specific amygdala activation states by different stressors.

Interestingly, it has been demonstrated that the exposure to a stressor may induce a form of metaplasticity that would affect the ability of a following stressor or of following BLA priming to modulate plasticity in other brain areas (Richter-Levin and Maroun, 2010). In relation to that Vouimba et al. (2004) found that while a single exposure to an elevated platform stress did not prevent LTP induction in the DG, it did induce a form of metaplasticity, since a repeated exposure to a similar stressor did suppress DG LTP. Thus, exposure to stress (Vouimba et al., 2004; Richter-Levin and Maroun, 2010) and amygdala priming (Richter-Levin and Maroun, 2010) were found to induce a form of metaplasticity that affects the ability of a following stress or following amygdala priming to modulate plasticity in other brain areas.

The current results further demonstrate that the BLA modulates synaptic plasticity in the hippocampus in a region-specific way (Vouimba and Richter-Levin, 2005; Vouimba et al., 2007). The nature of effects of BLA on synaptic plasticity differed between CA1 and the DG, such that in CA1 BLA activation impaired LTP, with stronger effects for stronger activation of the BLA, while in DG different stimulus intensities to the BLA resulted in differential effects on DG-LTP; a weak BLA stimulation enhanced DG-LTP but strong BLA stimulation impaired it. These results resemble those found following variant stressors, with a wide range of stressors found to suppress LTP in CA1 while different stressors found to affect DG-LTP on a spectrum from enhancement to impairment. Taken together, these findings lend further support to the notion that the BLA mediates some of the effects of stress and emotionality on hippocampal functioning (Richter-Levin, 2004).

\section{ACKNOWLEDGMENTS}

This research was supported by The Israel Science Foundation grant (no. 1403/07) to Gal Richter-Levin, and by the Institute for the Study of Affective Neuroscience, University of Haifa, which was endowed by the Hope for Depression Research Foundation. 


\section{REFERENCES}

Abe, K. (2001). Modulation of hippocampal long-term potentiation by the amygdala: a synaptic mechanism linking emotion and memory. Jpn. J. Pharmacol. 86, 18-22.

Abrahams, S., Morris, R. G., Polkey, C. E., Jarosz, J. M., Cox, T. C., Graves, M., and Pickering, A. (1999). Hippocampal involvement in spatial and working memory: a structural MRI analysis of patients with unilateral mesial temporal lobe sclerosis. Brain Cogn. 41, 39-65.

Ahmed, T., Frey, J. U., and Korz, V. (2006). Long-term effects of brief acute stress on cellular signaling and hippocampal LTP. J. Neurosci. 26, 3951-3958.

Akirav, I., and Richter-Levin, G. (1999a). Biphasic modulation of hippocampal plasticity by behavioral stress and basolateral amygdala stimulation in the rat. J. Neurosci. 19, 10530-10535.

Akirav, I., and Richter-Levin, G. (1999b). Priming stimulation in the basolateral amygdala modulates synaptic plasticity in the rat dentate gyrus. Neurosci. Lett. 270, 83-86.

Akirav, I., and Richter-Levin, G. (2002). Mechanisms of amygdala modulation of hippocampal plasticity. J. Neurosci. 22, 9912-9921.

Akirav, I., and Richter-Levin, G. (2006). Factors that determine the non-linear amygdala influence on hippocampus-dependent memory. Dose Response 4, 22-37.

Almaguer-Melian, W., Martinez-Marti, L., Frey, J. U., and Bergado, J. A. (2003). The amygdala is part of the behavioural reinforcement system modulating long-term potentiation in rat hippocampus. Neuroscience 119, 319-322.

Almaguer-Melian, W., Rojas-Reyes, Y., Alvare, A., Rosillo, J. C., Frey, J. U., and Bergado, J. A. (2005). Long-term potentiation in the dentate gyrus in freely moving rats is reinforced by intraventricular application of norepinephrine, but not oxotremorine. Neurobiol. Learn. Mem. 83, 72-78.

Berretta, S. (2005). Cortico-amygdala circuits: role in the conditioned stress response. Stress 8, 221-232.

Bliss, T. V., and Collingridge, G. L. (1993). A synaptic model of memory: long-term potentiation in the hippocampus. Nature 361, 31-39.

Bramham, C. R., Southard, T., Ahlers, S. T., and Sarvey, J. M. (1998). Acute cold stress leading to elevated corticosterone neither enhances synaptic efficacy nor impairs LTP in the dentate gyrus of freely moving rats. Brain Res. 789, 245-255.
Buffalari, D. M., and Grace, A. A. (2009). Anxiogenic modulation of spontaneous and evoked neuronal activity in the basolateral amygdala. Neuroscience 2163, 1069-1077.

Cahill, L. (2000). Neurobiological mechanisms of emotionally influenced, long-term memory. Prog. Brain Res. 126, 29-37.

Canli, T., Zhao, Z., Brewer, J., Gabrieli, J. D., and Cahill, L. (2000). Eventrelated activation in the human amygdala associates with later memory for individual emotional experience. J. Neurosci. 20, RC99.

Day, M., Langston, R., and Morris, R. G. (2003). Glutamate-receptormediated encoding and retrieval of paired-associate learning. Nature 424, 205-209.

De Kloet, E. R. (2004). Hormones and the stressed brain. Ann. N.Y. Acad. Sci. 1018, 1-15.

Diamond, D. M., Bennett, M. C., Stevens, K. E., Wilson, R. L., and Rose, G. M. (1990). Exposure to a novel environment interferes with the induction of hippocampal primed burst potentiation in the behaving rat. Psychobiology 18, 273-281.

Diamond, D. M., and Rose, G. M. (1994). Stress impairs LTP and hippocampal-dependent memory. Ann. N.Y. Acad. Sci. 746, 411-414.

Dunn, J. D., and Orr, S. E. (1984). Differential plasma corticosterone responses to hippocampal stimulation. Exp. Brain Res. 54, 1-6.

Eichenbaum, H., Schoenbaum, G., Young, B., and Bunsey, M. (1996). Functional organization of the hippocampal memory system. Proc. Natl. Acad. Sci. U.S.A. 93, 13500-13507.

Feldman, S., Conforti, N., and Siegel, R. A. (1982). Adrenocortical responses following limbic stimulation in rats with hypothalamic deafferentations. Neuroendocrinology 35, 205-211.

Foy, M. R., Stanton, M. E., Levine, S., and Thompson, R. F. (1987). Behavioral stress impairs long-term potentiation in rodent hippocampus. Behav. Neural. Biol. 48, 138-149.

Frey, S., Bergado-Rosado, J., Seidenbecher, T., Pape, H. C., and Frey, J. U. (2001). Reinforcement of early long-term potentiation (early-LTP) in dentate gyrus by stimulation of the basolateral amygdala: heterosynaptic induction mechanisms of late-LTP. J. Neurosci. 21, 3697-3703.

Garcia, R. (2001). Stress, hippocampal plasticity, and spatial learning. Synapse 40, 180-183.
Garcia, R., Musleh, W., Tocco, G., Thompson, R. F., and Baudry, M. (1997). Time-dependent blockade of STP and LTP in hippocampal slices following acute stress in mice. Neurosci. Lett. 233, 41-44.

Gelinas, J. N., and Nguyen, P. V. (2005). Beta-adrenergic receptor activation facilitates induction of a protein synthesis-dependent late phase of long-term potentiation. J. Neurosci. 25, 3294-3303.

Gerges, N. Z., Stringer, J. L., and Alkadhi, K. A. (2001). Combination of hypothyroidism and stress abolishes early LTP in the CA1 but not dentate gyrus of hippocampus of adult rats. Brain Res. 922, 250-260.

Gold, P. E., and van Buskirk, R (1978). Posttraining brain norepinephrine concentrations: correlation with retention performance of avoidance training and with peripheral epinephrine modulation of memory processing. Behav. Biol. 23, 509-520.

Goosens, K. A., and Maren, S. (2004). NMDA receptors are essential for the acquisition, but not expression, of conditional fear and associative spike firing in the lateral amygdala. Eur. J. Neurosci. 20, 537-548.

Hand, G. A., Hewitt, C. B., Fulk, L. J., Stock, H. S., Carson, J. A., Davis, J. M., and Wilson, M. A. (2002) Differential release of corticotropinreleasing hormone $(\mathrm{CRH})$ in the amygdala during different types of stressors. Brain Res. 949, 122-130.

Hirata, R., Matsumoto, M., Judo, C. Yamaguchi, T., Izumi, T., Yoshioka, M., and Togashi, H. (2009). Possible relationship between the stressinduced synaptic response and metaplasticity in the hippocampal CA1 field of freely moving rats. Synapse 63, 549-556.

Hurlemann, R., Matusch, A., Hawellek, B., Klingmuller, D., Kolsch, H., Maier, W., and Dolan, R. J. (2007a). Emotion-induced retrograde amnesia varies as a function of noradrenergic-glucocorticoid activity. Psychopharmacology (Berl) 194, 261-269.

Hurlemann, R., Wagner, M., Hawellek, B., Reich, H., Pieperhoff, P., Amunts, K., Oros-Peusquens, A M., Shah, N. J., Maier, W., and Dolan, R. J. (2007b). Amygdala control of emotion-induced forgetting and remembering: evidence from Urbach-Wiethe disease. Neuropsychologia 45, 877-884.

Ikegaya, Y., Saito, H., and Abe, K. (1995). High-frequency stimulation of the basolateral amygdala facilitates the induction of long-term potentiation in the dentate gyrus in vivo. Neurosci. Res. 22, 203-207.

Ikegaya, Y., Saito, H., and Abe, K. (1996). The basomedial and basolateral amygdaloid nuclei contribute to the induction of longterm potentiation in the dentate gyrus in vivo. Eur. J. Neurosci. 8 1833-1839.

Ilin, Y., and Richter-Levin, G. (2009). ERK2 and CREB activation in the amygdala when an event is remembered as "Fearful" and not when it is remembered as "Instructive". J. Neurosci. Res. 87, 1823-1831.

Izaki, Y., and Arita, J. (1996). Longterm potentiation in the rat hippocampal CAl region is inhibited selectively at the acquisition stage of discriminatory avoidance learning. Brain Res. 723, 162-168.

Izquierdo, I., and Medina, J. H. (1995). Correlation between the pharmacology of long-term potentiation and the pharmacology of memory. Neurobiol. Learn. Mem. 63, 19-32.

Katsuki, H., Izumi, Y., and Zorumski, C. F. (1997). Noradrenergic regulation of synaptic plasticity in the hippocampal CAl region. J. Neurophysiol. 77, 3013-3020.

Kavushansky, A., and Richter-Levin, G. (2006). Effects of stress and corticosterone on activity and plasticity in the amygdala. J. Neurosci. Res. 84 1580-1587.

Kavushansky, A., Vouimba, R. M., Cohen, H., and Richter-Levin, G. (2006). Activity and plasticity in the CAl, the dentate gyrus, and the amygdala following controllable vs uncontrollable water stress. Hippocampus 16, 35-42.

Kim, J. J., and Diamond, D. M. (2002) The stressed hippocampus, synaptic plasticity and lost memories. Nat. Rev. Neurosci. 3, 453-462.

Kim, J. J., Foy, M. R., and Thompson, R. F. (1996). Behavioral stress modifies hippocampal plasticity through N-methyl-D-aspartate receptor activation. Proc. Natl. Acad. Sci. U.S.A. 93, 4750-4753.

Kim, J. J., Koo, J. W., Lee, H. J., and Han, J. S. (2005). Amygdalar inactivation blocks stress-induced impairments in hippocampal long-term potentiation and spatial memory. J. Neurosci. 25, 1532-1539.

Kim, J. J., Lee, H. J., Han, J. S., and Packard, M. G. (2001). Amygdala is critical for stress-induced modulation of hippocampal long-term potentiation and learning. $J$. Neurosci. 21, 5222-5228.

Kim, J. J., and Fanselow, M. S. (1992). Modality-specific retrograde amnesia of fear. Science 256, 675-677. 
Kogan, I., and Richter-Levin, G. (2008). Activation pattern of the limbic system following spatial learning under stress. Eur. J. Neurosci. 27, 715-722.

Korol, D. L., and Gold, P. E. (2008). Epinephrine converts long-term potentiation from transient to durable form in awake rats. Hippocampus 18, 81-91.

Korz, V., and Frey, J. U. (2003). Stress-related modulation of hippocampal long-term potentiation in rats: Involvement of adrenal steroid receptors. J. Neurosci. 23, 7281-7287.

Korz, V., and Frey, J. U. (2005). Bidirectional modulation of hippocampal long-term potentiation under stress and no-stress conditions in basolateral amygdalalesioned and intact rats. J. Neurosci. 25, 7393-7400.

LaBar, K. S., and Cabeza, R. (2006). Cognitive neuroscience of emotional memory. Nat. Rev. Neurosci. 7, 54-64.

LeDoux, J. (2003). The emotional brain, fear, and the amygdala. Cell. Mol. Neurobiol. 23, 727-738.

LeDoux, J. E. (2000). Emotion circuits in the brain. Annu. Rev. Neurosci. 23, 155-184.

Li, Z., Zhou, Q., Li, L., Mao, R., Wang, M., Peng, W., Dong, Z., $\mathrm{Xu}, \mathrm{L}$., and Cao, J. (2005). Effects of unconditioned and conditioned aversive stimuli in an intense fear conditioning paradigm on synaptic plasticity in the hippocampal CA1 area in vivo. Hippocampus 15 , 815-824.

Lupien, S. J., Maheu, F., Tu, M., Fiocco, A., and Schramek, T. E. (2007). The effects of stress and stress hormones on human cognition: Implications for the field of brain and cognition. Brain Cogn. 65, 209-237.

Malenka, R. C., and Bear, M. F. (2004). LTP and LTD: an embarrassment of riches. Neuron 44, 5-21.

Malenka, R. C., and Nicoll, R. A. (1999). Long-term potentiation a decade of progress? Science 285, 1870-1874.

Maroun, M., and Akirav, I. (2008). Arousal and stress effects on consolidation and reconsolidation of recognition memory. Neuropsychopharmacology 33, 394-405.

Maroun, M., and Richter-Levin, G. (2003). Exposure to acute stress blocks the induction of long-term potentiation of the amygdalaprefrontal cortex pathway in vivo. J. Neurosci. 23, 4406-4409.

Martin, S. J., Grimwood, P. D., and Morris, R. G. (2000). Synaptic plasticity and memory: an evaluation of the hypothesis. Annu. Rev. Neurosci. 23, 649-711.

McGaugh, J. L. (2000). Memory a century of consolidation. Science 287, 248-251.

McGaugh, J. L. (2004). The amygdala modulates the consolidation of memories of emotionally arousing experiences. Annu. Rev. Neurosci. $27,1-28$.

Mesches, M. H., Fleshner, M., Heman, K. L., Rose, G. M., and Diamond, D. M. (1999). Exposing rats to a predator blocks primed burst potentiation in the hippocampus in vitro. J. Neurosci. 19, RC18.

Miyashita, T., and Williams, C. L. (2004). Peripheral arousalrelated hormones modulate norepinephrine release in the hippocampus via influences on brainstem nuclei. Behav. Brain Res. 153, 87-95.

Morris, R. G. (2001). Episodic-like memory in animals: psychological criteria, neural mechanisms and the value of episodic-like tasks to investigate animal models of neurodegenerative disease. Philos. Trans. R. Soc. Lond. B Biol. Sci. 356, 1453-1465.

Morris, R. G., Garrud, P., Rawlins, J. N., and O'Keefe, J. (1982). Place navigation impaired in rats with hippocampal lesions. Nature 297, 681-683.

Nakao, K., Matsuyama, K., Matsuki, N., and Ikegaya, Y. (2004). Amygdala stimulation modulates hippocampal synaptic plasticity. Proc. Natl. Acad. Sci. U.S.A. 101, 14270-14275.

Packard, M. G., and Cahill, L. (2001). Affective modulation of multiple memory systems. Curr. Opin. Neurobiol. 11, 752-756.

Pavlides, C., Nivon, L. G., and McEwen, B. S. (2002). Effects of chronic stress on hippocampal long-term potentiation. Hippocampus 12, 245-257.

Paxinos, G., and Watson, C. (1997). The Rat Brain in Stereotaxic Coordinates, 3rd Ed. New York, NY: Academic Press.

Pelletier, J. G., Likhtik, E., Filali, M., and Pare, D. (2005). Lasting increases in basolateral amygdala activity after emotional arousal: implications for facilitated consolidation of emotional memories. Learn. Mem. 12, 96-102.

Phelps, E. A. (2004). Human emotion and memory: interactions of the amygdala and hippocampal complex. Curr. Opin. Neurobiol. 14, 198-202.

Phelps, E. A., and LeDoux, J. E. (2005). Contributions of the amygdala to emotion processing: from animal models to human behavior. Neuron $48,175-187$.

Phillips, R. G., and LeDoux, J. E (1992). Differential contribution of amygdala and hippocampus to cued and contextual fear conditioning. Behav. Neurosci. 106, 274-285.

Prickaerts, J., and Steckler, T. (2005). "Effects of glucocorticoids on emotion and cognitive processes in animals," in Handbook of Stress and the Brain, eds T. S. Steckler, N. H. Kalin, and J. M. H. M Ruel (Amsterdam, The Netherlands: Elsevier), 359-385.

Richter-Levin, G. (2004). The amygdala, the hippocampus, and emotional modulation of memory. Neuroscientist 10, 31-39.

Richter-Levin, G., and Akirav, I. (2000) Amygdala-hippocampus dynamic interaction in relation to memory. Mol. Neurobiol. 22, 11-20.

Richter-Levin, G., and Maroun, M. (2010). Stress and amygdala suppression of metaplasticity in the medial prefrontal cortex. Cereb. Cortex 20, 2433-2441.

Roozendaal, B. (2000). (1999) Curt, P. Richter award. Glucocorticoids and the regulation of memory consolidation. Psychoneuroendocrinology 25, 213-238.

Roozendaal, B. (2002). Stress and memory: opposing effects of glucocorticoids on memory consolidation and memory retrieval. Neurobiol. Learn. Mem. 78, 578-595.

Roozendaal, B., McEwen, B. S., and Chattarii, S. (2009). Stress, memory and the amygdala. Nat. Rev. Neurosci. 10, 423-433.

Rubin, R. T., Mandell, A. J., and Crandall, P. H. (1966). Corticosteroid responses to limbic stimulation in man: localization of stimulus sites. Science 153, 767-768.

Sacchetti, B., Lorenzini, C. A., Baldi, E. Bucherelli, C., Roberto, M., Tassoni, G., and Brunelli, M. (2002). Timedependent inhibition of hippocampal LTP in vitro following contextual fear conditioning in the rat. Eur. J. Neurosci. 15, 143-150.

Seidenbecher, T., Reymann, K. G., and Balschun, D. (1997). A post-tetanic time window for the reinforcement of long-term potentiation by appetitive and aversive stimuli. Proc. Natl. Acad. Sci. U.S.A. 94, 1494-1499.

Shors, T. J., and Dryver, E. (1994). Effect of stress and long-term potentiation (LTP) on subsequent LTP and the theta burst response in the dentate gyrus. Brain Res. 666 , 232-238.

Shors, T. J., Gallegos, R. A., and Breindl, A. (1997). Transient and persistent consequences of acute stress on long-term potentiation (LTP), synaptic efficacy, theta rhythms and bursts in area CA1 of the hippocampus. Synapse 26, 209-217.

Shors, T. J., Seib, T. B., Levine, S., and Thompson, R. F. (1989). Inescapable versus escapable shock modulates long-term potentiation in the rat hippocampus. Science 244, 224-226.

Spyrka, J., and Hess, G. (2010) Repeated restraint-induced modulation of long-term potentiation in the dentate gyrus of the mouse. Brain Res. 1320, 28-33.

Squire, L. R., and Zola, S. M. (1996). Structure and function of declarative and nondeclarative memory systems. Proc. Natl. Acad. Sci. U.S.A 93, 13515-13522.

Thomas, M. J., Moody, T. D., Makhinson, M., and O'Dell, T. J. (1996). Activity-dependent beta-adrenergic modulation of low frequency stimulation induced LTP in the hippocampal CAl region. Neuron 17, 475-482.

Thompson, R. F., and Kim, J. J. (1996). Memory systems in the brain and localization of a memory. Proc. Natl. Acad. Sci. U.S.A. 93, 13438-13444.

Tsoory, M. M., Vouimba, R. M., Akirav, I., Kavushansky, A., Avital, A., and Richter-Levin, G. (2008). Amygdala modulation of memory-related processes in the hippocampus: potential relevance to PTSD. Prog. Brain Res. 167 35-51.

Vouimba, R. M., and Richter-Levin, G. (2005). Physiological dissociation in hippocampal subregions in response to amygdala stimulation. Cereb. Cortex 15, 1815-1821.

Vouimba, R. M., Yaniv, D., Diamond, D., and Richter-Levin, G. (2004). Effects of inescapable stress on LTP in the amygdala versus the dentate gyrus of freely behaving rats. Eur. J. Neurosci. 19, 1887-1894.

Vouimba, R. M., Yaniv, D., and RichterLevin, G. (2007). Glucocorticoid receptors and beta-adrenoceptors in basolateral amygdala modulate synaptic plasticity in hippocampal dentate gyrus, but not in area CA1. Neuropharmacology 52, 244-252.

Wang, J., Akirav, I., and RichterLevin, G. (2000). Short-term behavioral and electrophysiological consequences of underwater trauma. Physiol. Behav. 70, 327-332.

Watabe, A. M., Zaki, P. A., and O’Dell, T. J. (2000). Coactivation 
of beta-adrenergic and cholinergic receptors enhances the induction of long-term potentiation and synergistically activates mitogen-activated protein kinase in the hippocampal CA1 region. J. Neurosci. 20, 5924-5931.

Williams, C. L., Men, D., Clayton, E. C., and Gold, P. E. (1998). Norepinephrine release in the amygdala after systemic injection of epinephrine or escapable footshock: contribution of the nucleus of the solitary tract. Behav. Neurosci. 112, 1414-1422.

Xu, L., Anwyl, R., and Rowan, M. J. (1997). Behavioural stress facilitates the induction of long-term depression in the hippocampus. Nature 387, 497-500.

Yamada, K., McEwen, B. S., and Pavlides, C. (2003). Site and time dependent effects of acute stress on hippocampal long-term potentiation in freely behaving rats. Exp. Brain Res. 152, 52-59.

Yang, C. H., Huang, C. C., and Hsu, K. S. (2008). Differential roles of basolateral and central amygdala on the effects of uncontrollable stress on hippocampal synaptic plasticity. Hippocampus 18, 548-563.

Yarom, O., Maroun, M., and RichterLevin, G. (2008). Exposure to forced swim stress alters local circuit activity and plasticity in the dentate gyrus of the hippocampus. Neural Plast. 2008, 194097.

van Marle, H. J., Hermans, E. J., Qin, S., and Fernández, G. (2009). From specificity to sensitivity: how acute stress affects amygdala processing of biologically salient stimuli. Biol. Psychiatry 66, 649-655.

Conflict of Interest Statement: The authors declare that the research was conducted in the absence of any commercial or financial relationships that could be construed as a potential conflict of interest.
Received: 30 January 2012; accepted: 17 April 2012; published online: 04 May 2012.

Citation: $L i ~ Z$ and Richter-Levin G (2012) Stimulus intensity-dependent modulations of hippocampal long-term potentiation by basolateral amygdala priming. Front. Cell. Neurosci. 6:21. doi: 10.3389/fncel.2012.00021

Copyright (c) $2012 \mathrm{Li}$ and RichterLevin. This is an open-access article distributed under the terms of the Creative Commons Attribution Non Commercial License, which permits non-commercial use, distribution, and reproduction in other forums, provided the original authors and source are credited. 